\title{
A RHIC-style IPM in the Brookhaven AGS
}

\author{
R. Connolly, C. Dawson, J. Fite, H. Huang, \\ S. Jao, W. Meng, R. Michnoff, \\ P. Sampson, S. Tepikian
}

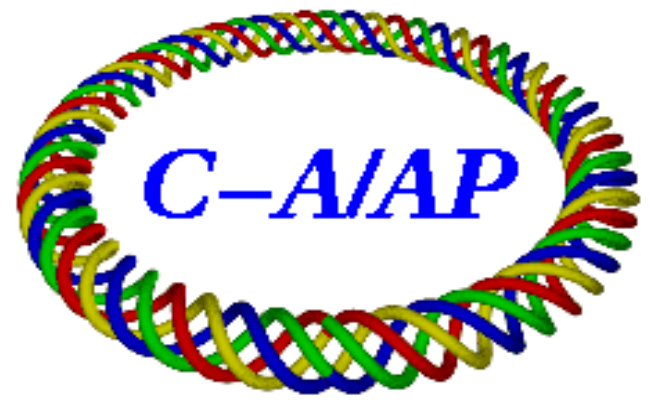

\section{Collider-Accelerator Department Brookhaven National Laboratory Upton, NY 11973}

Notice: This document has been authorized by employees of Brookhaven Science Associates, LLC under Contract No. DE-AC02-98CH10886 with the U.S. Department of Energy. The United States Government retains a nonexclusive, paid-up, irrevocable, world-wide license to publish or reproduce the published form of this document, or allow others to do so, for United States Government purposes. 


\title{
A RHIC-STYLE IPM IN THE BROOKHAVEN AGS*
}

\author{
R. Connolly, C. Dawson, J. Fite, H. Huang, S. Jao, W. Meng, \\ R. Michnoff, P. Sampson and S. Tepikian \\ Brookhaven National Lab, Upton, NY, USA
}

\section{Abstract}

Beam profiles in the two storage rings of the Relativistic Heavy-Ion Collider (RHIC) at Brookhaven National Lab (BNL) are measured with ionization profile monitors (IPMs). Each ring has a vertical and horizontal detector. An IPM measures the distribution of electrons produced in the beam line by beam ionization of background gas. These detectors have been developed at BNL in a program that began in 1996. The current detectors are a design that was based on a prototype built in 2007 and used in RHIC from 2007 to 2012. During the 2012 shutdown we refurbished this prototype and installed it into the Alternating-Gradient Synchrotron (AGS). This paper describes the new AGS IPM and shows data from the detector commissioning.

\section{INTRODUCTION}

The Relativistic Heavy-Ion Collider (RHIC) at Brookhaven National $\mathrm{Lab}$ is a pair of concentric synchrotrons in which counter-rotating beams intersect at six points [1]. Beams of ions from protons $\left(E_{\max }=250\right.$ $\mathrm{GeV})$ to fully-stripped uranium $\left(\mathrm{E}_{\max }=100 \mathrm{GeV} /\right.$ nucleon $)$ are accelerated and stored for several hours. There are detectors at two of the six intersection points for physics experiments with colliding beams.

Beam is injected into RHIC from a network of five accelerators. There are three primary accelerators: a tandem Van de Graaff, a 200-MeV H $\mathrm{H}^{-}$linac, and the new Electron-beam Ionization Source (EBIS). These three accelerators inject beam into the booster synchrotron then into the AGS which injects RHIC.

Ionization profile monitors (IPMs) have been developed at BNL to measure transverse beam profiles in RHIC [2]. An IPM measures the distribution of electrons in the beamline resulting from residual gas ionization during a bunch passage. The electrons are swept transversely from the beamline and collected on 64 strip anodes oriented parallel to the beam axis.

The current RHIC IPMs are copies of a prototype that was built in 2007 and used in RHIC until the 2012 shutdown. It was removed from RHIC in 2012, refurbished and installed in the AGS to measure the horizontal-beam profile.

*Work performed by employees of Brookhaven Science Associates, LLC under Contract No. DE-AC02-98CH10886 with the U.S. Department of Energy

\section{DETECTOR}

Figure 1 is a schematic of the detector and electronics in the accelerator tunnel, fig. 2 is a photo of the detector, and fig. 3 is the beam line installation. An electric field is placed across the $100 \mathrm{~mm} \times 150 \mathrm{~mm}$ rectangular beam pipe by biasing the top electrode at $-6 \mathrm{kV}$. The electrons are forced to travel perpendicularly to the measurement plane by a dipole magnetic field of $1.4 \mathrm{kG}$. A second, reversed, magnet corrects the AGS beam trajectory.

Electrons are swept towards a signal-gating grid which is normally biased at the sweep voltage of $-6 \mathrm{kV}$ to prevent the signal electrons from passing. To make measurements the grid is pulled to ground by a Behlke transistor switch which allows the signal electrons to pass through to the input of a microchannel plate (MCP). The MCP amplifies the electron flux by $10^{4}$ to $10^{7}$. Each channel of an MCP has a dead time of about $1 \mathrm{~ms}$ after firing. Periodically the signal electrons have to be gated off, with the MCP bias on, to allow the plate to recharge.

The amplified electron flux falls on an anode circuit board with 64 channels spaced by $0.53 \mathrm{~mm}$. Each channel is connected via vacuum feedthrough to one input of an eight-channel preamplifier board, fig. 4. Each input is ac coupled to a fast ac-coupled amplifier channel, which allow single-turn measurements of a bunch in AGS, and to a dc-coupled channel with $30 \mathrm{kHz}$ bandwidth. The amplifier outputs drive a shielded twisted-pair transmission line to a 10MSPS VME digitizer.

A screen-covered rectangular opening in the grounded half of the beam pipe decouples the electron gate from the beam. The collector board with attached MCP is located in a Faraday enclosure with an opening for the electrons covered by a grounded aluminum honeycomb material which attenuates rf by $80 \mathrm{~dB}$.

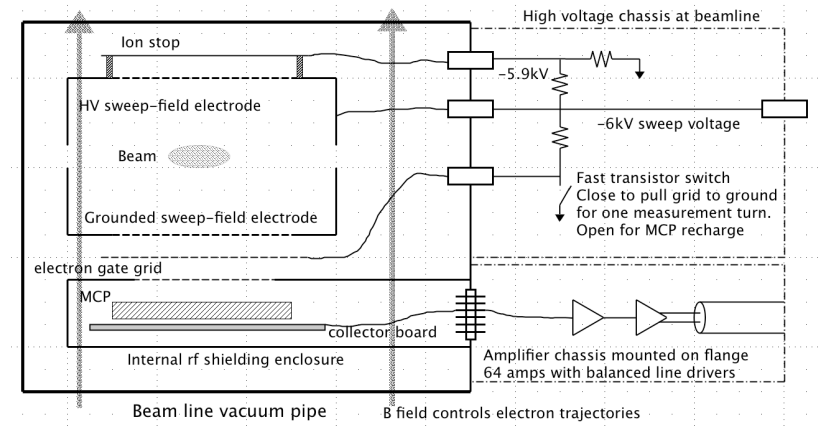

Figure 1: Schematic of detector and electronics located at the beamline. 


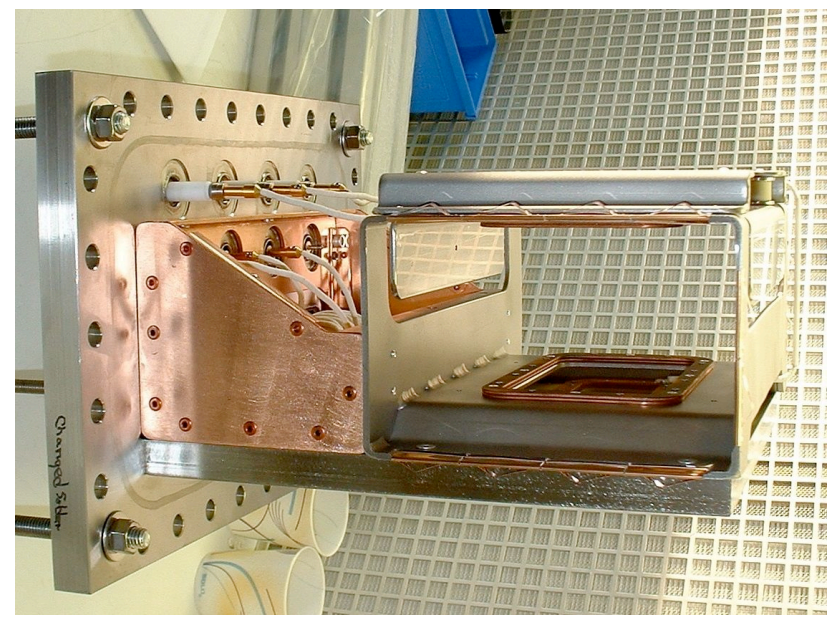

Figure 2. Assembled IPM transducer

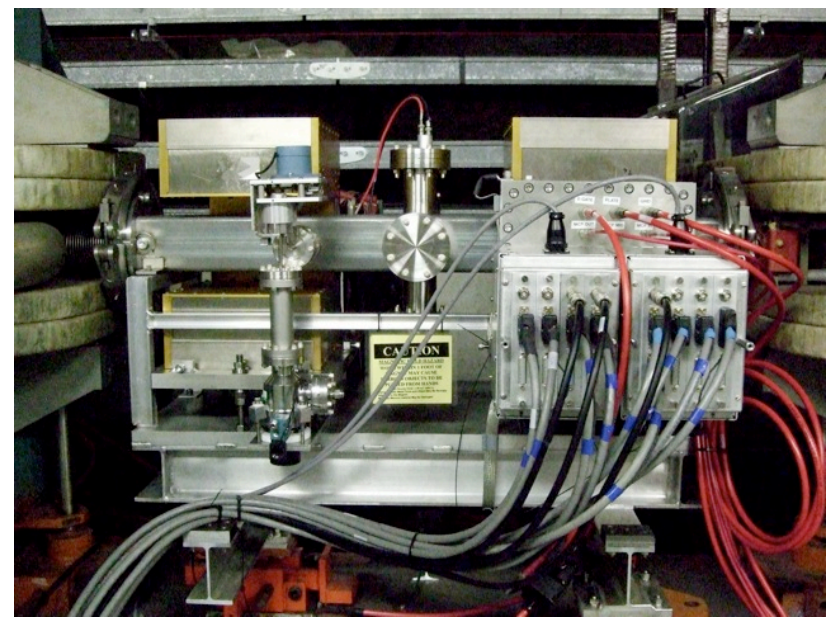

Figure 3. The detector installed in the beam line

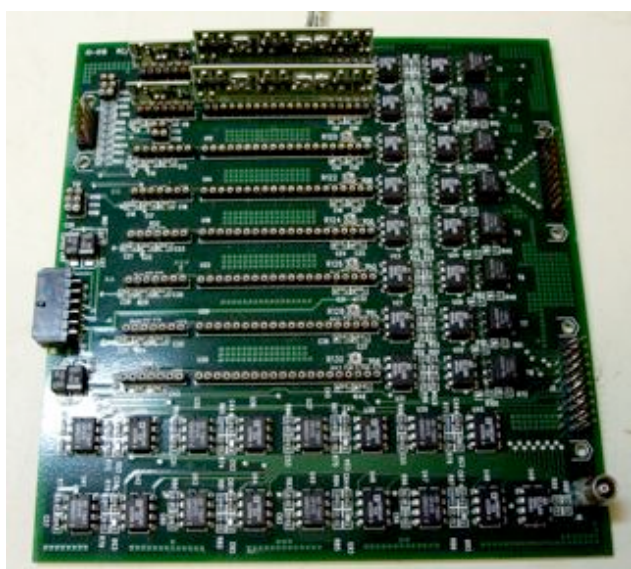

Figure 4. An 8-channel amplifier board. Two of the fast channels, top, are shown populated with the hybrid charge and shaper amps.

\section{DETECTOR COMMISSIONING}

The IPM hardware was not ready before the the 2013 beam run started so it was installed on maintenance days during the run. The beam line hardware, fig. 4, was installed in early April. During limited tunnel access periods we installed the electronics and terminated cables. Since this was an installation in a new machine we encountered many unanticipated challenges. We obtained the first complete beam profile in late May and on June 4 we measured the profile in fig. 5.

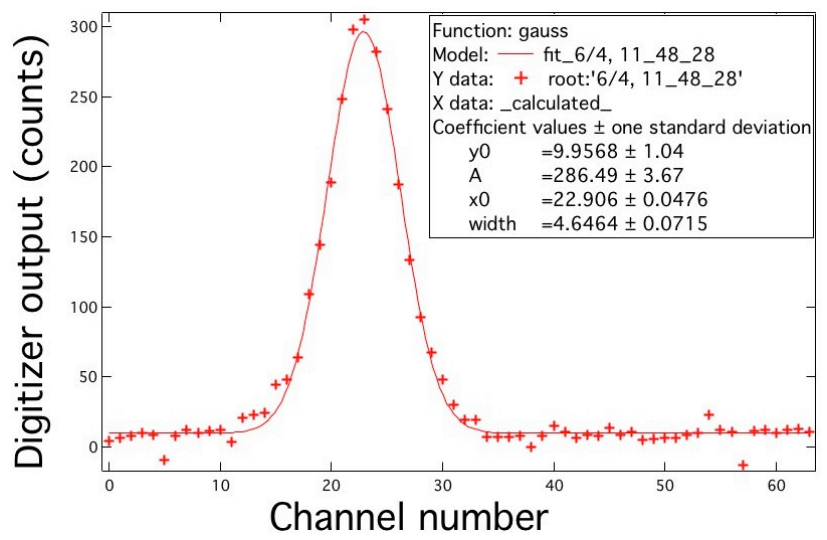

Figure 5. Beam profile during the acceleraion ramp

One known challenge is horizontal orbit stability in the AGS. All events in the AGS cycle are timed from the $\mathrm{t}_{0}$ event. Beam injection is at $143 \mathrm{~ms}$, the energy ramp ends at $580 \mathrm{~ms}$, and extraction is at $1.4 \mathrm{~s}$ after $\mathrm{t}_{0}$. During the cycle the beam moves around horizontally over a range up to $6 \mathrm{~cm}$. Since the IPM measurement aperture is $3.4 \mathrm{~cm}$, the beam is out of this aperture much of the time.

The profile in fig. 5 was taken at $t_{0}+425 \mathrm{~ms}$. After injection the beam was outside of the measurement aperture until about $375 \mathrm{~ms}$, about halfway up the ramp. Figure 6 shows the results of a series of measurements taken at delays of $375 \mathrm{~ms}$ to $1.2 \mathrm{~s}$. A Gaussian was fitted to each measurement. The red crosses show the beam sigmas and the blue circles show the beam centers.

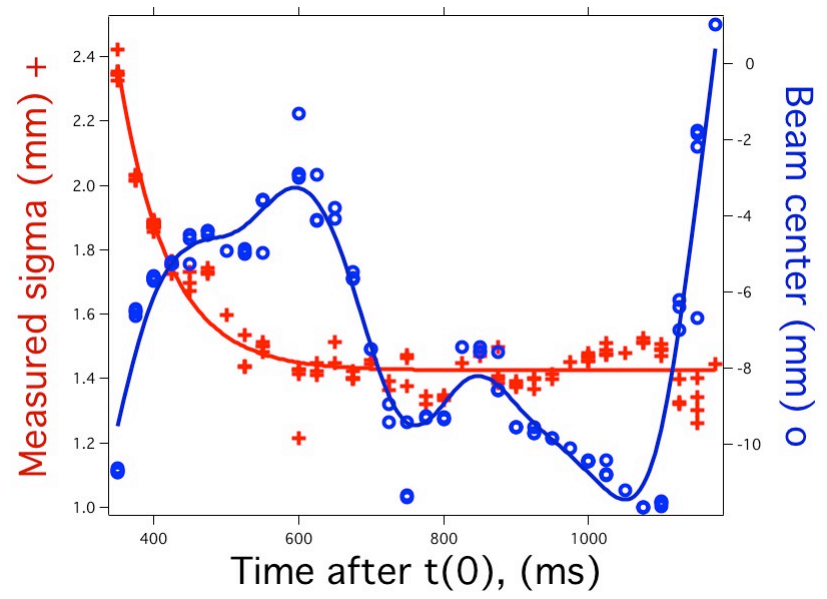

Figure 6. Beam widths (red +) and beam center (blue o) from second half of acceleration ramp to extraction 
The measurement sequence in fig. 6 starts about halfway up the acceleration ramp. When commissioning started the beam at injection was outside of the IPM measurement aperture and moved into the aperture at about $375 \mathrm{~ms}$.

After the operators centered the beam at injection, we took a data set in which we measured the bunch profile on the first 700 turns after injection over 109 consecutive machine cycles. We averaged these 109 measurements to produce a mountain-range plot of the bunch profile on the first 700 turns after injection. Figure 7 shows turns 300700. There is an amplitude and width oscillation of period 61 turns which is exponentially damping with time constant of 327 turns. Figure 8 shows the profiles amplitudes (red + ) and widths (blue o) fitted with exponentially-damped sine waves.

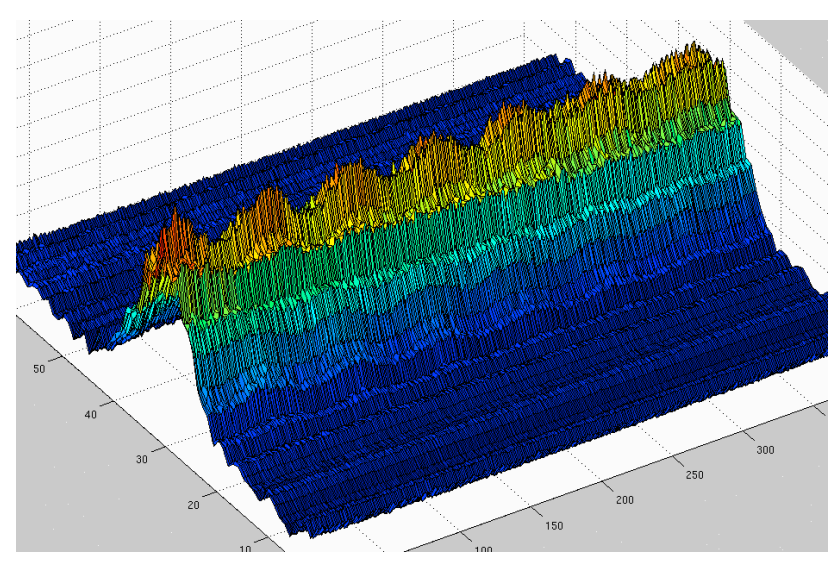

Figure 7. Mountain-range of turns 300-700 after injection. Channels 1-64 are front to back.

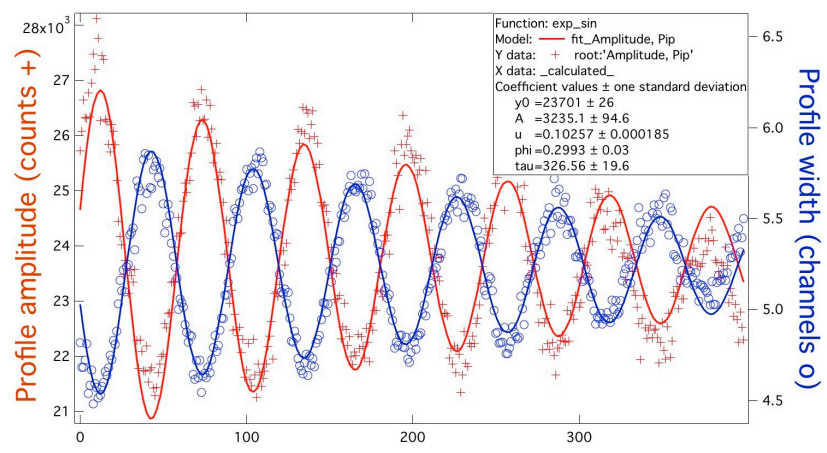

Turn number (zero is 300 turns after injection)

Figure 8. Profile amplitudes (red + ) and widths (blue o) of turns 300-700 after injection.

\section{DISCUSSION}

We installed a RHIC IPM into the AGS to measure horizontal beam profiles. The vacuum chamber was a modified RHIC chamber. Our goal was to demonstrate the device would work, make basic commissioning measurements and identify AGS-specific operation challenges.

\section{REFERENCES}

[1] http://www.rhic.bnl.gov/

[2]

http://accelconf.web.cern.ch/AccelConf/BIW2010/PAPER S/TUPSM010.PDF 